\section{El consumo de drogas en el debate de la salud pública}

\author{
Drug use in the public health debate \\ O consumo de drogas no debate da saúde pública
}

Andrés Felipe Tirado-Otálvaro 1,2

\title{
Resumen
}

Este texto sitúa el consumo de drogas ilegales en el debate actual entre la salud pública tradicional, frente a las propuestas latinoamericanas, y propone la necesidad de abordar el tema desde de una salud pública alternativa, centrada en la vida y el protagonismo de los sujetos, los grupos y los movimientos sociales, mediante una praxis contrahegemónica, para orientar así la discusión sobre la necesidad de generar políticas públicas incluyentes y democráticas. El consumo de drogas ha sido abordado desde la medicina clínica como un problema que genera trastornos mentales y enfermedades infecciosas, ya sea por las prácticas sexuales de riesgo, o el uso de jeringas para el consumo intravenoso; desde la política, como un problema relacionado con fenómenos delictivos; $y$ desde la salud pública tradicional, como un problema que genera abandono escolar y laboral, aumento en la demanda de servicios de salud, muerte y violencia; sin embargo, no todas las formas de consumo representan un uso problemático, ni desencadenan trastornos por uso de sustancias.

Política de Salud; Estigma Social; Trastornos Relacionados con Sustancias; Drogas Ilícitas

\author{
1 Universidad Pontificia \\ Bolivariana, Medellín. \\ Colombia. \\ 2 Universidad de Antioquia, \\ Medellín, Colombia. \\ Correspondencia \\ A. F. Tirado-Otálvaro \\ Universidad Pontificia \\ Bolivariana. \\ Calle 78B\#72A-109, Medellín \\ felipe.tirado@upb.edu.co
}




\section{Postura de la salud pública tradicional}

Las diferentes formas en que se presenta el consumo de drogas no necesariamente representan un uso problemático de las mismas, como fenómenos delictivos, desintegración familiar, desvinculación académica o laboral, desarrollo de enfermedades mentales, transmisión de enfermedades infecciosas, intoxicación y muerte 1 , ya que el consumo se puede dar de manera experimental, ocasional, habitual o compulsiva 2 .

El consumo experimental se caracteriza por presentarse de manera fortuita, durante un periodo de tiempo muy limitado o en cantidades reducidas, así que la droga se puede probar una o varias veces, pero después no se vuelve a consumir; el consumo ocasional se presenta de manera intermitente, en cantidades a veces importantes, y su principal motivación es la integración grupal, mejorar el rendimiento deportivo, académico o aumentar el goce sexual 2,3. A diferencia de los dos anteriores, el consumo habitual se realiza a diario, con el fin de aliviar el malestar psicoorgánico que se presenta tras su uso (síndrome de abstinencia) o para mantener el rendimiento normal del individuo, el consumidor habitual dedica gran parte del día a pensar en la droga y en obtenerla; por último, el consumo compulsivo se presenta de manera muy intensa y varias veces al día, lo que da lugar a un trastorno del comportamiento con múltiples consecuencias afectivas, laborales, académicas, familiares y sociales 2,3 .

La dependencia a drogas puede ser física o psicológica; la primera se caracteriza por la tolerancia (disminución gradual de los efectos de la droga tras la administración repetida, lo cual obliga a incrementar la dosis para alcanzar los mismos efectos) y el síndrome de abstinencia (signos y síntomas agudos que se presentan tras la suspensión del consumo); mientras que la dependencia psicológica se caracteriza por el deseo intenso y ansiedad de consumo, al igual que por el efecto reforzador positivo (vivencias placenteras, de evasión o de aprobación por pares) o por el refuerzo negativo (uso de drogas para evitar los efectos tras la suspensión del consumo) 2,4. Se debe aclarar que se puede presentar dependencia física o psicológica, sin que haya lugar a un diagnóstico clínico de trastorno por uso de sustancias (TUS); ya que para que esta situación se presente es necesaria la evaluación médica, por parte de un experto que determine un patrón problemático de consumo de la sustancia, que lleve a discapacidad clínicamente significativa en un periodo de 12 meses o más a partir de los criterios establecidos en la quinta versión del Manual Diagnóstico y Estadístico de Trastornos Mentales (DSM-V) 5 .
El texto pone en debate el consumo de drogas ilegales desde las posturas de la salud pública tradicional, frente a las propuestas teóricas latinoamericanas, por medio de la interpretación que cada una de estas corrientes hace del concepto de salud; al igual que por los métodos que emplean para acercarse a la realidad y la práctica salubrista que ejecutan. Esta revisión teórica constituye un avance en la construcción del proyecto de tesis doctoral del autor, y pretende ofrecer una contribución al estudio del fenómeno de las drogas desde la propuesta de Edmundo Granda de una salud pública alternativa, centrada en la vida y el protagonismo de los sujetos, los grupos y los movimientos sociales por medio de una praxis contrahegemónica, para orientar la discusión sobre la necesidad de generar políticas públicas incluyentes, equitativas, democráticas y saludables.

El modelo biomédico dominante que ha puesto un especial interés en lo biológico sobre lo sociocultural 6 , tiene como su máximo representante a la medicina clínica, la cual centra su saber y sus métodos alrededor de la enfermedad y la muerte 7; sin embargo, entender la salud en términos mecanicistas y organicistas, como los planteados por Descartes ${ }^{8}$, o expresarla simplemente como la ausencia de la enfermedad, es una reducción en función de lo normal y estadísticamente típico ${ }^{9}$. La salud es inmensurable y ubicar a las personas en patrones de normalidad es enfermar a la población 10 .

Disciplinas derivadas del positivismo como la medicina y la epidemiología clínica, clínica entienden el consumo de drogas y los TUS como un problema de salud pública mundial, y resaltan los efectos nocivos que dichas sustancias tienen para la salud, los cuales incluyen trastornos de salud física y mental, aumento en la demanda de servicios de salud, altos índices de incapacidad escolar y laboral, fenómenos delictivos y propagación de epidemias como el VIH, la hepatitis B y C 11,12. A pesar de esto, homologar el consumo experimental u ocasional de drogas ilegales con los TUS y considerarlos como categorías análogas, desconoce el componente recreativo del uso de dichas sustancias y genera situaciones de discriminación, estigmatización y exclusión 13.

El concepto de estigma, es utilizado para definir diferentes situaciones vergonzosas según los términos que para una comunidad son tolerables o no, de acuerdo al conjunto de normas y valores aceptados por una sociedad; en tal sentido la medicina clínica, hace referencia al estigma como desviación, haciendo hincapié en la definición social del comportamiento anormal, o aquello indeseable que merece ser descalificado; es así como los consumidores de drogas ilegales 
al ser estigmatizados terminan siendo rechazados socialmente 1,13 .

El estigma es el resultado de un proceso que involucra cinco componentes interrelacionados entre sí. En el primero hay un etiquetamiento de las personas debido a sus diferencias; en el segundo, la persona etiquetada es estereotipada de acuerdo a características indeseables; en el tercero hay una separación entre ellos y nosotros; en el cuarto, debido al etiquetamiento como drogadicto o enfermo, se pierde la condición de sujeto y aparece la discriminación, la devaluación, el rechazo y la exclusión; por último, en el quinto componente se involucra el ejercicio del poder 14 .

La estigmatización y la discriminación como procesos culturales y sociales, al estar relacionadas con conceptos de poder y dominación, se encuentran necesariamente ligadas a los estudios de desigualdad 13, entendida esta última por Breilh como expresión resultante de inequidad (categoría que define las relaciones de poder, producto de la apropiación y acumulación económica, política y cultural por parte de las clases dominantes) 15 . Lo anterior adquiere una enorme importancia en términos de salud pública y justicia social, ya que la discriminación y la exclusión a la que se ven sometidos los consumidores de drogas al ser etiquetados como enfermos; es decir, como pacientes con TUS, limita sus posibilidades de acceder a derechos fundamentales como la salud, la educación y el trabajo 14; según Mario Hernández-Álvarez 16, el valor central para definir una desigualdad como inequidad es la justicia, de manera que la inequidad es una desigualdad considerada injusta.

Los discursos estigmatizantes que homologan el consumo recreativo de drogas ilegales con los TUS, tienen una función de distorsión y magnificación, ya que el uso de dichas sustancias ha sido socialmente asociado al crimen, al peligro que representan los consumidores para la sociedad y a la enfermedad, lo que se traduce en la cosificación del fenómeno y la invisibilización del sujeto 13. La marginación de ciertos sectores de la población, al igual que la escasa atención que se presta a las voces de las minorías, o los grupos vulnerables al momento de identificar e intervenir los problemas, junto con el evidente distanciamiento entre el Estado y la sociedad civil, se presentan como importantes limitaciones de la salud pública tradicional para dar respuesta a fenómenos complejos 17 .

El debate sobre el estigma que ha impuesto el etiquetaje de los consumidores experimentales $\mathrm{u}$ ocasionales de drogas ilegales como "drogadictos”, es relevante en términos de salud pública, dada la importancia del reconocimiento del suje- to desde sus múltiples dimensiones y potencialidades, como un ser no solamente biológico; sino también, sociocultural, psicoafectivo e histórico, que se encuentra en una relación dialéctica con la sociedad 18. En otras palabras, se trata de una dialéctica desde un punto de vista ético, entre el sujeto moral y el sujeto histórico-político, en la cual se pretende asumir al ser humano como un fin en sí mismo, y no como un medio, en el marco del respeto recíproco y la intersubjetividad 16 .

\section{Limitaciones de la salud pública tradicional para entender el fenómeno de las drogas}

El trípode sobre el cual se fundamenta la salud pública tradicional, tiene como presupuesto filosófico-teórico a la enfermedad y la muerte para explicar la salud; como presupuesto metodológico, se centra en el método positivista para explicar la verdad y el riesgo; y como presupuesto praxeológico, detenta el poder en el Estado para asegurar la prevención de la enfermedad 7 . A continuación, se presentan las limitaciones que dichos presupuestos tienen para entender el fenómeno de las drogas.

\section{Limitaciones desde el presupuesto teórico- filosófico de la salud pública tradicional}

Ante los grandes logros alcanzados por la medicina clínica sobre la enfermedad individual, se construyó el discurso de la salud pública hegemónica para dar cuenta de la enfermedad colectiva o pública, como sumatoria de enfermedades particulares 19. Esta óptica hipotetiza sobre los sujetos desde una base poblacional, ubicando de manera paralela el discurso para "todos los individuos", "todos los usuarios de drogas" y "todos los drogodependientes", lo cual desconoce la subjetividad, invisibiliza la dimensión social del fenómeno y deja a las diferentes colectividades sin rostro 13,20 .

La salud pública tradicional considera desde su posición funcionalista a la población como objetos, más que como sujetos, y hace una lectura de la realidad por medio de la razón tecnológica e instrumental; motivo por el cual, sus métodos presentan importantes limitaciones para dar respuesta a situaciones propias de una realidad cambiante y compleja 17. De ahí que desde esta postura, se planteen como opciones para el fenómeno de las drogas, la penalización como respuesta punitiva al delito que representa el uso de éstas, y la medicalización, como respuesta terapéutica a la representación que se hace del consumo como enfermedad; desconociendo que 
el problema en cuestión no lo representa "la droga”, sino los significados que a nivel social se le dan a estas sustancias 13 .

La medicalización consiste en definir un problema en términos médicos, a partir del uso de un lenguaje técnico para su descripción, utilizando una intervención médica para "tratar" el problema en cuestión 21. La lógica dicotómica del pensamiento hegemónico en salud pública, y su concepción de la enfermedad como anormalidad o desviación, ha traído consigo la medicalización como principal estrategia de control a la transgresión de la norma, situación que ha sido particularmente evidente en el tema del uso y dependencia de drogas ilegales 13 .

El modelo biomédico dominante sobre el cual se soportan la mayoría de las políticas públicas, individualiza situaciones colectivas y descontextualiza los problemas sociales 21 , en tanto los presupuestos funcionalistas que los soportan, ahorran a la salud pública su preocupación por el sujeto individual y colectivo 19. La medicalización puede tener serias implicaciones para la justicia social en una sociedad altamente estratificada, en tanto para los tomadores de decisiones, es mucho más práctico inclinarse por soluciones médicas que por medidas económicas, políticas o sociales 21 .

Limitaciones desde el presupuesto metodológico de la salud pública tradicional

El positivismo científico sobre el cual está soportado el presupuesto metodológico de la salud pública tradicional 7, encontró en los modelos casualistas de la disciplina epidemiológica occidental desde mediados del siglo XX, la mejor herramienta para abordar y explicar la realidad, ya fuese desde de la tríada ecológica de la enfermedad de Leavell-Clark, o desde las redes de multicausalidad de MacMahon 22, siempre bajo la premisa de entender la exposición como algo externo y el riesgo como un fenómeno contingente, debido a su visión antropocéntrica, individualista, biologicista y fragmentada de la realidad 23 .

La epidemiología clínica ha podido explicar cómo la unión de varios factores puede aumentar la vulnerabilidad en los individuos respecto al uso de drogas ilegales, y cómo esto puede generar predisposición favorable al consumo 24,25; es así como desde el enfoque positivista, dicha disciplina puede explicar cómo algunas variables personales (baja autoestima, trastornos del comportamiento, escasa capacidad de afrontamiento, consumo por curiosidad o imitación) y sociales (disponibilidad y acceso a las sustancias, problemas del funcionamiento familiar y relación con otros consumidores) se comportan como factores de riesgo para el consumo y dependencia de drogas 11,24,26,27,28,29. Sin embargo, el enfoque positivista al hacer énfasis en la enfermedad, y al considerar la población como la suma de individuos, desconoce al sujeto y al componente social, cultural, político e histórico del asunto en cuestión, motivo por el cual no logra dar respuesta a las problemáticas de una situación compleja 19,30,31.

Limitaciones desde el presupuesto praxeológico de la salud pública tradicional

El presupuesto práctico de la salud pública tradicional le otorga un especial reconocimiento al poder del Estado, como el encargado de asegurar la prevención de la enfermedad 19, en tanto éste organiza las acciones preventivas y apoya la gestión de los servicios de atención médica sobre la población 7 .

La salud pública dominante, con el soporte argumentativo del enfoque de riesgo de la epidemiología, opera a favor del interés estratégico de los poderosos y desde allí sustenta su hegemonía ${ }^{15}$. Desde esta perspectiva, es posible entender como el discurso de la eficiencia económica en la oferta de bienes y servicios de salud, la focalización de acciones preventivas y la medicalización de la enfermedad han configurado la propuesta ofensiva del Estado neoliberal para la atención en salud 22 .

El fenómeno de las drogas no ha sido ajeno a la discusión arriba planteada; es así como el discurso del crimen, asociado al uso de sustancias ilegales y el etiquetamiento de peligrosidad, con el cual son representados los consumidores, han sido la clave para argumentar la penalización y la medicalización; no sólo desde la estigmatización, sino también desde las diferentes estructuras de poder que argumentan el control público e impiden el goce de los derechos sociales, económicos y culturales, bajo el fundamento de la salud orquestado por el discurso moralista de la ciencia médica dominante 13,21.

La acumulación de capital, propia del modelo neoliberal actual, interviene en diferentes espacios institucionales, técnicos y académicos, los cuales son funcionales al poder, minando los lugares de discusión e impidiendo el pensamiento crítico en salud 15. El capitalismo técnico e instrumental favorece la exclusión, la criminalidad y produce una profunda crisis de autoridad y legitimidad que debilita el carácter soberano de los Estados 7 y aumenta la inequidad 15 . 


\section{Posturas de la salud pública latinoamericana}

Como crítica a los modelos antropocéntricos y biologicistas hegemónicos, que explican la realidad desde el enfoque positivista, surgen en América Latina diferentes corrientes teóricas en contraposición al pensamiento científico dominante 6 . A continuación se presenta una síntesis de los movimientos de la medicina social latinoamericana, salud colectiva y salud pública alternativa, para posteriormente identificar cómo desde el pensamiento latinoamericano se entiende el proceso salud enfermedad, y la praxis de la salud pública, uniendo lo anterior al estudio del fenómeno de las drogas.

\section{Medicina social latinoamericana}

La medicina social latinoamericana, que retoma algunos aspectos del pensamiento anglosajón de la medicina social de Inglaterra, Alemania y Francia del siglo XIX, tiene como sus principales exponentes a Juan César García, María Isabel Rodríguez, Asa Cristina Laurell y Jaime Breilh 17, quienes hacen uso del pensamiento MarxistaNeo Marxista en el campo de la salud 32. Particularmente Laurell y Breilh, soportan sus tesis en el materialismo crítico, la economía política y la ecología política, en diálogo con una versión crítica de las ciencias de la salud, el ambiente y la sociedad 33, para comprender el proceso saludenfermedad como un acontecimiento humano y social, mediado por determinantes históricos y macrosociales, en el que intervienen categorías como los procesos de producción, la clase social 17 , los modos de vida, la etnia y la raza, en comunicación dialéctica con los procesos de emancipación social 22.

Jaime Breilh, uno de los más importantes exponentes la epidemiología crítica latinoamericana (eje disciplinar del movimiento de la medicina social latinoamericana), argumenta que las condiciones socioeconómicas de la estructura social, son las principales determinantes para enfermar y morir, por lo cual propone que desde la investigación se debe asumir una posición militante en cuanto a la movilización social y política para afectar los determinantes estructurales o socioeconómicos de los procesos de reproducción social 34 .

\section{Salud colectiva}

La corriente brasileña de la salud colectiva que tiene como sus principales exponentes a Naomar Almeida Filho, Jairnilson Silva Paim y Gastão Wagner de Sousa Campos, entre otros, recoge algunos de los postulados de la medicina social latinoamericana; sin embargo, mientras que esta última centra su acción en las necesidades derivadas de los procesos estructurales de determinación, la salud colectiva busca la articulación de diferentes enfoques científicos para abordar el complejo salud-enfermedad-atención, sin menospreciar la historicidad e integralidad de cada enfoque 17.

El objeto de estudio de la salud colectiva se construye en los límites de lo biológico y de lo social, donde confluyen diferentes disciplinas para superar el biologicismo dominante, la naturalización de la vida social y la subordinación al modelo médico hegemónico 35 , a partir de la vinculación de categorías epistemológicas como la complejidad, modelos teóricos como la teoría del caos, y formas lógicas de análisis, como los modelos matemáticos no lineales, la geometría fractal, la lógica borrosa y la teoría de redes 36,37. En tal sentido, la salud colectiva propone intervenir en las formas de distribución de poder, los cambios de comportamiento, la cultura, las instituciones, la producción de conocimiento y la organización y regulación de los recursos y procesos productivos, por medio de la acción conjunta del Estado y el compromiso de la sociedad para la producción de ambientes y poblaciones saludables, a través de actividades profesionales generales y especializadas 35 .

\section{Salud pública alternativa}

La salud pública alternativa desde la postura teórica planteada por Granda; el cual retoma a autores como Varela y Maturana 17, se fundamenta en las metáforas de la vida, el conocimiento y el buen poder político, en contraposición a los presupuestos teórico-filosóficos de la enfermedad y la muerte; el método positivista como presupuesto metodológico para explicar la verdad y el riesgo; y el poder del Estado para asegurar la prevención de la enfermedad, como presupuesto praxeológico de la salud pública hegemónica 7,19.

La salud pública alternativa formula una reflexión sobre la salud desde aproximaciones filosóficas diferentes a la dominante, en tanto no centra su objeto de estudio en la enfermedad; ni su praxis en los métodos propuestos por la epidemiología, ni la administración en salud tradicional 7. Esta corriente alternativa aboga por una doble hermenéutica entre los grupos sociales y el aporte de la ciencia; la primera hermenéutica se hace de manera directa con la población, con el objetivo de comprender sus verdades, eticidades, prácticas y estrategias de organización del poder, mientras que la segunda se hace con el aporte de la ciencia y la técnica con miras a enriquecer las formas de vida cotidiana de los grupos sociales 38 . 


\section{Abordaje del consumo de drogas ilegales desde una posición discursiva diferente a los supuestos de salud y salud pública planteados por la ciencia dominante}

Una postura teórica diferente a la tradicional debe entender la salud como una forma de vida autónoma y solidaria, consustancial a la cultura, dependiente y condicionante de las relaciones que se establecen con la naturaleza, la sociedad y el Estado 7.

El discurso del modelo biomédico hegemónico, desde la perspectiva dicotómica en la que aborda la salud y la enfermedad, ubica a los consumidores de drogas ilegales como enfermos, desviados o anormales 1,13; pero la salud no pertenece al orden de los promedios, ni es el resultado de tablas comparativas 39 , por lo que expresar la salud en términos de normalidad estadística, no permite explicar las representaciones que se alejan del patrón común 40 .

Cada individuo es libre y responsable de las decisiones que tome respecto a su salud; en tal sentido, Cangilhem plantea que la posibilidad de abusar de la salud forma parte esta misma 41 . La salud es la capacidad de romper las normas impuestas y construir nuevas normas bajo los requerimientos de adaptación de un mundo versátil 30; no se trata entonces dominar la enfermedad como lo pretende el enfoque positivista 10, puesto que salud se debe comprender desde la vida misma, y no sólo desde el cálculo del riesgo que ocurre antes de que el cuerpo enferme ${ }^{42}$. El concepto de salud va más allá de la capacidad de autocuidado, la seguridad contra los riesgos, la capacidad para corregir ese margen de tolerancia y los medios para enfrentar las dificultades 43; la salud es un punto de encuentro entre lo biológico y lo social 44 , que incluye realidades como las de la cultura, el lenguaje, el pensamiento, el comportamiento y la sociedad 40 .

A diferencia de lo planteado por la salud pública tradicional, un grupo social no es la suma de sus individuos; lo que define a un grupo social son las relaciones que se establecen históricamente entre sus miembros y las formas de reproducción social que lo caracterizan 40 . El proceso salud-enfermedad trasciende al individuo, ya que abarca aspectos culturales, sociales, económicos y políticos 43; visto de otro modo, es un conjunto de posibilidades para la adaptación a la vida social y de grupo por parte de los individuos 40 .

Así como la medicina clínica deja de lado el saber de los sujetos, y los supedita a someterse a sus dictámenes científicos; la epidemiología clásica suprime la diversidad cultural y la histo- ricidad del proceso salud-enfermedad, al centrarse en la razón instrumental que fundamenta el método científico 7. En contraposición con lo anterior, una concepción de salud centrada en la vida, rescata al sujeto en la salud pública desde una perspectiva histórica-sociocultural-ontológica; es decir, desde la dialéctica entre el sujeto y la sociedad 18 .

El uso de drogas forma parte de la cultura, tanto como el lenguaje o el trabajo, y es inherente la historia de la humanidad 45 , y aunque los efectos nocivos que tienen las drogas para la salud han sido ampliamente descritos en los textos médicos de toxicología y psiquiatría; en el microcosmos en el que se desenvuelve la realidad de los consumidores, los significados que éstos le dan a las drogas pueden interpretarse como situaciones meramente recreativas, terapéuticas o placenteras. En tal sentido Restrepo plantea que cuando se consume una droga, no solamente se está consumiendo una sustancia química en sí misma, sino que se está consumiendo el sistema valorativo que la acompaña 45 , motivo por el cual una salud pública alternativa centrada en la vida, debe propender por el reconocimiento del sujeto y la subjetividad 38 .

El ser humano orienta sus acciones a partir de los significados que le otorga a las diferentes situaciones con las que se encuentra 46 , y aunque las decisiones sobre políticas públicas en el tema de drogas han sido influenciadas tradicionalmente por argumentos lógicos-racionales; los principios morales de los tomadores de decisiones juegan un papel importante en términos de políticas sociales, ya que si éstos le otorgan a las drogas un significado relacionado con el crimen o la enfermedad, lo anterior se verá reflejado en el ámbito de las decisiones políticas que tomen con relación al asunto en cuestión 47 .

Cuando se aborda el consumo de drogas con el velo de la moral, la medicina clínica y el derecho penal; se invisibiliza el sujeto y se desconoce el componente histórico del fenómeno, ya que al excluir del discurso el poder terapéutico y recreativo de las drogas, se olvida también que éstas producen sensaciones de bienestar, placer, euforia, relajación, generan empatía y apertura emocional hacia otras personas 26,48,49,50,51,52,53 Es por esto, que desde una postura teórica diferente a la hegemónica, el centro no estaría en la enfermedad y "la droga", sino en las definiciones sociales que se producen en el vínculo entre los sujetos y las drogas, las cuales incluyen los significados sociales y culturales que subyacen detrás del consumo 13 .

Una salud pública alternativa centrada en la vida, como la propuesta por Granda, no desecha lo diverso; por el contrario, surge desde lo 
local, comprende lo complejo y tiene respeto por la cultura, la temporalidad y la historia 7; valora la dimensión subjetiva de las prácticas de salud, las vivencias de los sujetos y propicia la reflexión en salud al generar espacios de comunicación y diálogo con otros saberes 18. Es necesaria la descategorización, al igual que suprimir etiquetamiento y la estigmatización de los consumidores experimentales y ocasionales de drogas como “drogadictos", "enfermos” o "delincuentes", como un primer paso para la generación de políticas públicas saludables en este tema; para esto se requiere otorgarle protagonismo y voz a las culturas no científicas, a las culturas invisibilizadas y las culturas particulares 54 .

La salud pública alternativa propone un giro filosófico-teórico desde la comprensión de la salud (entendida como enfermedad y muerte), hacia la interpretación del proceso salud-enfermedad como salud y vida; un giro metodológico desde el método positivista-explicativo, limitado a la razón instrumental y la epidemiología, como disciplina que lo acredita, hacia el método hermenéutico-comprensivo (centrado en la doble hermenéutica entre el saber colectivo y el saber científico de diferentes disciplinas que aportan a la comprensión del complejo saludenfermedad); y un giro práctico desde el protagonismo del Estado (como fuerza privilegiadora para calcular el riesgo y asegurar la prevención), al coprotagonismo de los sujetos, los grupos y los movimientos sociales, para liberar el pensamiento y la acción en salud del bloqueo producido por el desarrollo capitalista, por medio de una praxis transformadora que presione al Estado y los poderes dominantes para la construcción de políticas públicas saludables, equitativas y democráticas 7,19,54,55.

\section{Conclusiones}

El consumo recreativo de drogas ilegales no representa un uso problemático de las mismas; sin embargo, el etiquetamiento de los consumidores experimentales u ocasionales como drogadictos o delincuentes, genera discriminación, exclusión y rechazo, lo que refuerza procesos de subjetivación, como baja autoestima, falta de confianza en las propias capacidades y visión fatalista del futuro, que no sólo inciden en la autorregulación con relación al consumo de drogas; sino que también, favorece la exclusión de los sujetos de espacios socio-afectivos, y de espacios de integración social 1, y limita posibilidad de entender la salud como capacidad para corregir ese margen de tolerancia y buscar los medios para enfrentar las dificultades 43 .
El estigma que produce el consumo de drogas ilegales sobre sus usuarios opera de manera negativa sobre las opciones que éstos tienen en términos de acceso y permanencia a un empleo digno y educación formal, así como a diferentes servicios sociales y beneficios públicos, lo que limita las posibilidades de superación y dificulta las perspectivas de resiliencia y reintegración social 1. Poner en discusión las categorías que se asocian al consumo de drogas ilegales como enfermedad y delito, y diferenciar a los consumidores recreativos de los usuarios problemáticos, constituye uno de los asuntos más urgentes con relación al fenómeno de las drogas a nivel mundial en términos salud pública y justicia social 13 .

El fenómeno de las drogas se ha estudiado ampliamente desde enfoques empírico-analíticos a nivel mundial, a través de diversas investigaciones que han incluido grandes grupos poblacionales, para hacer ya sea descripciones o análisis de causalidad, como lo demuestran los estudios más recientes al respecto $1,56,57,58$. Sin embargo, aspectos subjetivos como el placer, raramente son incluidos en los debates públicos sobre el tema, en tanto el enfoque metodológico hegemónico ha eliminado del discurso la corporalidad, lo histórico y lo cultural por considerarlos temas frívolos o poco científicos. Las consecuencias que esto ha traído en términos de investigación son devastadoras y se pueden evidenciar en el claro distanciamiento entre el saber científico y el saber colectivo, lo cual trasciende de igual modo, la manera como se formulan las políticas públicas en el tema 59 .

La comprensión integral del fenómeno de las drogas, amerita métodos de investigación que no desconozcan los procesos históricos, biológicos y sociales que rodean dicha situación; lo anterior implica el respeto a la complejidad de los objetos y la pluralidad de los diferentes enfoques científicos, para la comprensión de modos alternativos de intervención de un problema interdisciplinario ${ }^{9}$. En tal sentido, entender la salud y la enfermedad como proceso, genera un reto adicional a la investigación, ya que resalta el papel protagónico de las ciencias sociales en salud, sin desconocer la importancia de la investigación clínica y epidemiológica 40; por lo cual, el enfoque positivista tradicional que pretende develar verdades universales, deberá dialogar con propuestas que consideren verdades particulares y diversas 30 .

Abordar el consumo de drogas desde el agente, a partir de sus discursos; permite comprender el fenómeno desde sí mismo, desde su significado, mucho más allá del simple relato de sucesos o la enumeración de acontecimientos 60; este diálogo con el sujeto permite generar conocimientos desde el punto de vista teórico que se pueden 
orientar a mejorar procesos de intervención y generación de políticas incluyentes, en tanto favorecen el discurso intersubjetivo con el otro; esto significa poder comprender el mundo en el que él vive para traducirlo a través de esta dialéctica en procesos sociales mediados por la interacción entre los diferentes actores 46 .

Es necesario abordar el fenómeno de las drogas en consonancia con políticas que enfrenten las múltiples formas de exclusión social como la falta de voz pública, la falta de ingresos para satisfacer necesidades básicas, la falta de empleo formal y la falta de acceso a servicios de salud, entre otros; generando espacios críticos de debate, no sólo con la academia, sino también con los sujetos, las comunidades y los movimientos sociales 1 . En concordancia con lo anterior, Almeida Filho \& Paim 35 destacan la importancia de interpretar la salud y la vida a través de lógicas recursivas y aproximaciones ontológicas que privilegian el conocimiento, el aprendizaje y la acción de cambio.

La participación del Estado en términos de inversión social, educación, salud e investigación es un punto clave; sin embargo, este proceso no será efectivo, si no involucra además la movilización y el empoderamiento social por la reivindicación de su derecho a la salud y el respeto por la diferencia. La fuerza para alcanzar la salud se encuentra en las mismas poblaciones; por dichas razones no es posible confiar en que sólo con la intervención de la ciencia positiva y el paternalismo del Estado se alcance dicha condición 42 .

Es innegable el papel protagónico que las drogas han tenido en la política, la religión, la ciencia, el arte, el deporte, el medio ambiente, las leyes, la economía y la guerra, ya que su uso forma parte de la historia de la humanidad 45 , de ahí la importancia de reconocer el proceso histórico, dinámico, cultural y social que subyace tras el consumo; sin juzgarlo desde perspectivas criminalizantes y represivas, en tanto el objetivo es comprender el fenómeno de las drogas como un asunto de salud pública y no sólo como un asunto moral 47,61.

Las políticas en el tema de drogas que se diseñan desde perspectivas que entienden a la población como la sumatoria de individuos, y que centran sus discursos en la enfermedad, la represión y la penalización; no diferencian el consumo recreativo del uso problemático, ni reconocen la complejidad del fenómeno. En tal sentido la generación de políticas públicas sobre el tema requiere no solamente un conjunto de acciones aisladas, sino, también, el reconocimiento de métodos investigativos diferentes al dominante la consideración de sus resultados en el debate público, el respeto por la diferencia y la movilización social.

Investigar el fenómeno de las drogas desde una salud pública alternativa, como la propuesta por Granda, supone un reto para la práctica de esta disciplina y la generación de nuevo conocimiento, orientado al diseño de políticas públicas que consideren el saber colectivo, los movimientos sociales, la diversidad y lo complejo, así como métodos de investigación diferentes al tradicional, que favorezcan el debate crítico y permitan enriquecer la práctica con miras a presionar los poderes dominantes en la construcción de políticas públicas incluyentes, equitativas y democráticas.

\section{Agradecimientos}

Al Comité para el Desarrollo de la Investigación (CODI), por su estrategia de sostenibilidad de 2013 a 2014, de la Universidad de Antioquia para la financiación de productos académicos.

\section{Referencias}

1. Organización de los Estados Americanos. El problema de las drogas en las Américas. Washington DC: Organización de los Estados Americanos; 2013.

2. Martín M, Ladero JM, Lizasoain I. Conceptos fundamentales en drogodependencia. In: Lorenzo P, Ladero JM, Leza JC, Lizasoain I, editores. Drogodependencias: farmacología, patología, psicología legislación. Madrid: Editorial Médica Panamericana; 2009. p. 1-24. 
3. Castaño G. Historia natural de la enfermedad adictiva. In: Velásquez de P. E, Olaya Pérez A, Castaño Pérez G, Castro Rey S, editores. Adicciones, aspectos clínicos y psicosociales, tratamiento y prevención. Medellín: Fondo Editorial CIB; 2013. p. 77-87.

4. Maureira F. comparación de las características neurales y conductuales. Rev Chil Neuropsicol 2009; 4:84-90.

5. American Psychiatric Association. Diagnostic and statistical manual of mental disorders. Fifth edition. DSM-5. Arlington: American Psychiatric Association; 2013.

6. Franco Á. Tendencias y teorías en salud pública. Rev Fac Nac Salud Pública 2006; 24:119-30.

7. Granda E. La salud pública y las metáforas sobre la vida. Rev Fac Nac Salud Pública 2000; 18:83-100.

8. Descartes R. Meditaciones metafísicas. Quito: Libresa; 1973.

9. Almeida Filho N. For a General Theory of Health: preliminary epistemological and anthropological notes. Cad Saúde Pública 2001; 17:753-99.

10. Gadamer H-G. El estado oculto de la salud. Barcelona: Editorial Gedisa; 1996.

11. Lakarra S, López de Jesús M, Meana Martínez JJ. Bases biológicas, psicológicas y socioculturales. In: Pérez J, Valderrama J, Cervera G, Rubio G, editores. Tratado SET de trastornos adictivos. Madrid: Editorial Médica Panamericana; 2006. p. 1-13.

12. Leza JC, Ladero JM, Lizasoain I. Drogodependencias: farmacología, patología, psicología, legislación. Madrid: Editorial Médica Panamericana; 2003.

13. Vázquez A, Stolkiner A. Procesos de estigma y exclusión en salud: articulaciones entre estigmatización, derechos ciudadanos, uso de drogas y drogodependencia. Anu Investig-Fac Psicol Univ B Aires 2009; 16:295-303.

14. Link BG, Phelan JC. Stigma and its public health implications. Lancet 2006; 367:528-9.

15. Breilh J. Las tres 'S' de la determinación de la vida: 10 tesis hacia una visión crítica de la determinación social de la vida y la salud. In: Nogueira RP, editor. Determinação social da saúde e reforma sanitária. Rio de Janeiro: Centro Brasileiro de Estudos de Saúde; 2010. p. 87-125.

16. Hernández-Álvarez M. El concepto de equidad y el debate sobre lo justo en salud. Rev Salud Pública 2008; 10 Suppl 1:72-82.

17. Valencia Arredondo M. Aportes de los nuevos enfoques para la conformación de la salud pública alternativa. Rev Fac Nac Salud Pública 2011; 29: 85-93.

18. Peñaranda C. Salud pública y justicia social en el marco del debate determinantes -determinación social de la salud. Rev Fac Nac Salud Pública 2013; 31 Suppl 1:S91-102.

19. Granda E. A qué llamamos salud colectiva, hoy. Rev Cuba Salud Pública 2004; 30(2). http://scielo. sld.cu/scielo.php?script=sci_arttext\&pid=S0864 34662004000200009\&lng=es.

20. Conrad P. Sobre la medicalización de la anormalidad y el control social. In: Ingleby D, editor. Psiquiatría crítica: la política de la salud mental. Barcelona: Editorial Crítica; 1982. p. 129-59.
21. Conrad P. Medicalization and social control. Annu Rev Sociol 1992; 18:209-32.

22. Breilh J. Una perspectiva emancipadora de la investigación y acción, basada en la determinación social de la salud. In: Taller Latinoamericano sobre Determinantes Sociales de la Salud: documento para la discusión. México DF: Asociación Latinoamericana de Medicina Social; 2008. p. 14-29.

23. Breilh J. Epidemiología crítica: ciencia emancipadora e interculturalidad. Buenos Aires: Lugar Editorial; 2003.

24. Silva LV, Malbergier A, Stempliuk VA, Andrade AG. Factors associated with drug and alcohol use among university students. Rev Saúde Pública 2006; 40:280-8.

25. Dirección Seccional de Salud de Antioquia; Instituto de Ciencias de la Salud CES; Empresa Social del Estado CARISMA. Prevalencia de consumo de sustancias psicoactivas y factores asociados: resultados de la investigación en jóvenes escolarizados del Departamento de Antioquia 2003. Medellín: Dirección Seccional de Salud de Antioquia; Instituto de Ciencias de la Salud CES; Empresa Social del Estado CARISMA; 2003.

26. Pérez J, Valderrama J, Cervera G, Rubio G, editores. Tratado SET de trastornos adictivos. Madrid: Editorial Médica Panamericana; 2006.

27. Del Moral M, Fernández L. Fundamentos psicosociales de las drogodependencias: prevención y tratamiento. In: Lorenzo P, Ladero JM, Leza JC, Lizasoain I, editores. Drogodependencias: farmacología, patología, psicología, legislación. Madrid: Editorial Médica Panamericana; 2009. p. 27-62.

28. Wilens TE, Fusillo S. When ADHD and substance use disorders intersect: relationship and treatment implications. Curr Psychiatry Rep 2007; 9:408-14.

29. Wilson JJ. ADHD and substance use disorders: developmental aspects and the impact of stimulant treatment. Am J Addict 2007; 16:5-13.

30. Granda E. Salud pública e identidad. In: Ponencias: el Foro Modelos de Desarrollo, Espacio Urbano y Salud. Santa Fe de Bogotá: Alcaldía Mayor de Santa Fe de Bogotá/Organización Panamericana de la Salud; 1999

31. Franco S. Proceso vital humano-proceso salud enfermedad: una nueva perspectiva. In: Seminario Marco Conceptual para la Formación de Profesionales de la Salud. Bogotá: Universidad Nacional de Colombia; 1993.

32. Martinez FJM, Silva LR. La Medicina Social latinoamericana y sus miradas múltiples sobre la investigación cualitativa. Nueva Época 2004; 9: 39-46.

33. Breilh J. La determinación social de la salud como herramienta de transformación hacia una nueva salud pública (salud colectiva). Rev Fac Nac Salud Pública 2013; 31 Suppl 1:S13-27.

34. Hernández LJ. Que critica la epidemiología crítica: una aproximación a la mirada de Naomar Almeida Filho. Boletín del Observatorio en Salud 2009; 2:18-28.

35. Almeida Filho N, Paim JS. La crisis de la salud pública y el movimiento de salud colectiva en Latinoamérica. Cuad Méd Soc (Ros.) 1999; (75):5-30. 
36. Almeida-Filho N. Complejidad y transdisciplinariedad en el campo de la salud colectiva: evaluación de conceptos y aplicaciones. Salud Colect 2006; 2:123-46.

37. Ramis Andalia RM, Sotolongo Codina PL. Aportes del pensamiento y las ciencias de la complejidad al estudio de los determinantes de la salud. Rev Cuba Salud Pública 2009; 35:65-77.

38. Granda E, editor. La salud y la vida. Quito: Organización Panamericana de la Salud; 2009.

39. Canguilhem G. La salud, concepto vulgar y problema filosófico. Revista Sociología 1998; 21:151-8.

40. Quevedo E. El proceso salud-enfermedad: hacia una clínica y una epidemiología no positivistas. In: Ponencias: Seminario Permanente Salud y Administración. Bogotá: Postgrado en Administración en Salud, Facultad de Estudios Interdisciplinarios, Universidad Javeriana; 1990.

41. Canguilhem G. Lo normal y lo patológico. México DF: Siglo XXI; 1986.

42. Granda E. Salud, ambiente y desarrollo: un reencuentro con los temas fundamentales de la salud pública. In: Ponencias: V Congreso Internacional de Salud Pública. Medellín: Universidad de Antioquia; 2007.

43. Caponi S. Georges Canguilhem and the epistemological status of the concept of health. Hist Ciênc Saúde-Manguinhos 1997; 4:287-307.

44. Frenk J. La nueva salud pública. In: Organización Panamericana de la Salud, editor. La crisis de la salud pública: reflexiones para el debate. Washington DC: Organización Panamericana de la Salud; 1992. p. 75-83. (Publicación Científica, 540).

45. Restrepo LC. Dimensiones culturales del consumo de psicoactivos. In: Serres M, editor. ¿Legalizar la droga? Seis escritos sobre el tema. Medellín: Corporación Región; 1994. p. 41-8.

46. Berger P, Luckmann T. La construcción social de la realidad. Buenos Aires: Amorrortu; 1986.

47. Avendaño M. Las políticas de drogas: la reducción de daños en relación con otros modelos teóricos. In: Castaño Pérez G, editor. Nuevas estrategias de atención en drogodependencias: la reducción de riesgos y daños. Medellín: Fondo Editorial FUNLAM; 2001. p. 273-85.

48. Fernández J, Ramos A. Cannabis. In: Pérez J, Valderrama J, Cervera G, Rubio G, editores. Tratado SET de trastornos adictivos. Madrid: Editorial Médica Panamericana; 2006. p. 222-7.
49. Navarro M, Ladero JM, Martín M, Leza JC. Complicaciones orgánicas y psiquiátricas. In: Lorenzo P, Ladero JM, Leza JC, Lizasoain I, editores. Drogodependencias: farmacología, patología, psicología, legislación. Madrid: Editorial Médica Panamericana; 2009. p. 329-58.

50. Moro M, Leza JC. Opioides I. Farmacología. Intoxicación aguda. In: Lorenzo P, Ladero JM, Leza JC, Lizasoain I, editores. Drogodependencias: farmacología, patología, psicología, legislación. Madrid: Editorial Médica Panamericana; 2009. p. 107-26.

51. Flores A. Cocaína: bases biológicas de la dependencia de Cocaína. In: Pérez J, Valderrama J, Cervera G, Rubio G, editores. Tratado SET de trastornos adictivos. Madrid: Editorial Médica Panamericana; 2006. p. 242-51.

52. Vallejo J, Leal C. Tratado de psiquiatría. Barcelona: Ars Médica; 2005.

53. Bobes J, Sáiz Martínez PA, García-Portilla González MP. Alucinógenos, Anfetaminas y Drogas de Síntesis: epidemiología, clínica y tratamiento. In: Pérez J, Valderrama J, Cervera G, Rubio G, editores. Tratado SET de trastornos adictivos. Madrid: Editorial Médica Panamericana; 2006. p. 188-93.

54. Granda E. El saber en salud pública en un ámbito de pérdida de antropocentrismo y ante una visión de equilibrio ecológico. In: Granda E, editor. La salud y la vida. Quito: Organización Panamericana de la Salud; 2009. p. 187-224.

55. Granda E. El sujeto, la ética y la salud. In: Granda E, editor. La salud y la vida. Quito: Organización Panamericana de la Salud; 2009. p. 97-110.

56. Organización de los Estados Americanos. Estrategia hemisferica sobre drogas 2010. Washington DC: Organización de los Estados Americanos; 2010.

57. United Nations Office on Drugs and Crime. World drug report 2010. Washington DC: United Nations; 2010.

58. United Nations Office on Drugs and Crime. World drug report 2012. Washington DC: United Nations; 2012.

59. Moore D. Erasing pleasure from public discourse on illicit drugs: on the creation and reproduction of an absence. Int J Drug Policy 2008; 19:353-8.

60. Ricoeur P. La vida: un relato en busca de narrador. Ágora - Papeles de Filosofía 2006; 25:9-22.

61. Serres M. Drogas. In: Serres M, editor. ¿Legalizar la droga? Seis escritos sobre el tema. Medellín: Corporación Región; 1994. p. 7-12. 


\section{Abstract}

This article addresses illegal drug use within the current debate in traditional public health and in proposals from Latin America, while emphasizing the need to approach the issue from an alternative public health perspective centered on individual users, groups, and social movements as protagonists. This counterhegemonic approach thus aims to orient the discussion on the need for inclusive and democratic public policies. Illegal drug use has been addressed from various perspectives: clinical medicine, viewing it as a problem that generates mental disorders and infectious diseases, both through risky sexual practices and/or use of injecting paraphernalia; from a legal perspective, as a problem related to delinquency; and according to traditional public health, as a problem that generates school dropout and work absenteeism and increases the demand on health services, in addition to increasing violence and death. However, not all forms of drug consumption involve problematic use, nor do they all trigger disorders related to substance use.

Health Policy; Social Stigma; Substance-Related Disorders; Street Drugs

\section{Resumo}

Este texto contextualiza o consumo de drogas ilegais no debate da saúde pública tradicional, frente às propostas latino-americanas, e sugere a necessidade de se abordar a questão a partir de uma saúde pública alternativa, centrada na vida e no papel dos indivíduos, grupos e movimentos sociais, mediante uma práxis contra-hegemônica, orientaando assim a discussão sobre a necessidade de geração de políticas públicas inclusivas e democráticas. $O$ uso de drogas foi abordado a partir: da medicina clínica, como um problema que gera transtornos mentais e doenças infecciosas, tanto por sexo inseguro, quanto pelo uso de seringas para uso intravenoso; da política, como um problema relacionado com fenômenos criminais; e da saúde pública tradicional, como um problema que gera abandono escolar e de trabalho, aumento da demanda por serviços de saúde, morte e violência. No entanto, nem todas as formas de consumo representam um uso problemático ou desencadeam transtornos por uso de substâncias.

Política de Saúde; Estigma Social; Transtornos

Relacionados ao Uso de Substâncias; Drogas Ilícitas
Recibido el 27/Oct/2015

Versión final presentada el 14/Mar/2016 Aprobado el 06/Abr/2016 